\title{
ON THE FUNDAMENTAL SOLUTION FOR THE REAL MONGE-AMPÈRE OPERATOR
}

\author{
ZBIGNIEW BŁOCKI ${ }^{1}$ and JOHAN THORBIÖRNSON
}

\section{Abstract.}

We discuss the basic properties of the fundamental solution for the real Monge-Ampère operator, like continuity and symmetry. We also give formulas for the solution in the unit ball and product domains.

\section{Introduction.}

For smooth convex functions $u$ the Monge-Ampère operator is defined by

$$
M u:=\operatorname{det}\left(\frac{\partial^{2} u}{\partial x_{j} \partial x_{k}}\right) .
$$

It turns out that one can well define $M u$ to be a nonnegative Borel measure for an arbitrary convex function $u$. There are at least two different but equivalent ways of doing it. The first one is the geometric approach which was developped by Alexandrov and Bakelman in the 50's and 60's. The starting point is the following observation: if $u$ is smooth and strictly convex in a convex domain $\Omega$ then

$$
\int_{E} \operatorname{det}\left(\frac{\partial^{2} u}{\partial x_{j} \partial x_{k}}\right) d \lambda=\lambda(\nabla u(E)), \quad E \text { Borel subset of } \Omega
$$

where $\lambda$ is the $n$-dimensional Lebesgue measure. Now one defines the right hand-side of $(0.1)$ for an arbitrary convex function $u$. The gradient image of a point $x_{0} \in \Omega$ is the set of all $p \in \mathrm{R}^{n}$ with

$$
u\left(x_{0}\right)+\left\langle x-x_{0}, p\right\rangle \leq u(x), \quad x \in \Omega .
$$

(Here $\langle\cdot, \cdot\rangle$ denotes the scalar product in $\mathrm{R}^{n}$.) In fact the equation $y=u\left(x_{0}\right)+\left\langle x-x_{0}, p\right\rangle$ defines a supporting hyperplane to the graph of $u$ at $x_{0}$ and it is obvious that if $u$ is smooth at $x_{0}$ then the gradient image of $x_{0}$

\footnotetext{
${ }^{1}$ Partially supported by KBN Grant No. 2 PO3A 05809 and the Foundation for Polish Science (FNP) scholariship.

Received June 20, 1996.
} 
consists of exactly one point $\nabla u\left(x_{0}\right)$. Now we can define $\nabla u(E)$, the gradient image of the set $E$, to be the union of the gradient images of all points belonging to $E$. By a theorem of Alexandrov the set of points which belong to the gradient image of more than one point of $\Omega$ has Lebesgue measure zero which means that the union is almost disjoint. One shows that $M u(E):=\lambda(\nabla u(E))$ is a Borel measure (see [13] for the details). The second way to define $M u$ is to use the theory of the complex Monge-Ampère operator. The latter one is defined by

$$
M_{c} u:=\operatorname{det}\left(\frac{\partial^{2} u}{\partial z_{j} \partial \bar{z}_{k}}\right)
$$

if $u$ is a smooth plurisubharmonic (psh) function in an open subset of $\mathrm{C}^{n}$. There is no counterpart of (0.1) in the complex case. However, as shown by Bedford and Taylor [2] (see also [11]), $M_{c} u$ can be defined as a nonnegative Borel measure for all continuous psh functions in such a way that $M_{c} u_{j}$ converges weakly to $M_{c} u$ if $u_{j}$ converges uniformly to $u$. This determines $M_{c} u$ uniquely since every continuous psh function can be locally uniformly approximated by smooth psh functions. Now if $u$ is convex in $\Omega \subset \mathrm{R}^{\mathrm{n}}$ then it can be regarded as a psh function of $\Omega+i \mathrm{R}^{n} \subset \mathrm{C}^{n}$. Then one can easily show that $M_{c} u$ defines a measure in $\Omega$ which is actually $4^{-n} M u$ (see also [13]).

If $\Omega$ is a bounded domain in $\mathrm{R}^{\mathrm{n}}$ then it is natural to consider the following Dirichlet problem

$$
\left\{\begin{array}{l}
u \in \operatorname{CVX}(\Omega) \cap \mathrm{C}(\bar{\Omega}) \\
M u=\mu \\
\left.u\right|_{\partial \Omega}=f .
\end{array}\right.
$$

Here $\mu$ is a nonnegative Borel measure in $\Omega$ and $f \in \mathrm{C}(\partial \Omega)$. The uniqueness of (0.2) follows from the following comparison principle: if $u, v \in \operatorname{CVX}(\Omega) \cap \mathrm{C}(\bar{\Omega})$ are such that $M u \geq M v$ in $\Omega$ and $u \leq v$ on $\partial \Omega$ then $u \leq v$ on $\bar{\Omega}$. On the other hand (0.2) has a solution if $\Omega$ is strictly convex and $\mu(\Omega)<\infty$ (we refer to [13] for the details). This can be used to show that (0.2) has a unique solution if $\Omega$ is just convex, $\mu(\Omega)<\infty$ and $f=0$ (see [3], Theorem 4.1).

The aim of this paper is to study the fundamental solution of the MongeAmpère operator $M$. Let $\Omega$ be bounded and convex and take $y \in \Omega$. Then there is a unique solution of the following Dirichlet problem

$$
\left\{\begin{array}{l}
u \in \mathrm{CVX}(\Omega) \cap \mathrm{C}(\bar{\Omega}) \\
M u=\delta_{y} \\
\left.u\right|_{\partial \Omega}=0 .
\end{array}\right.
$$

Define $g_{\Omega}(x, y):=u(x)$ and $h_{\Omega}(y):=g_{\Omega}(y, y)$. It follows from the comparison 
principle that $u$ is affine along the intervals joining $y$ with the boundary. Therefore $h_{\Omega}(y)$ determines $g_{\Omega}(\cdot, y)$ and that is why we are mostly concerned with the function $h_{\Omega}$. One can easily show that

$$
g_{\Omega}(x, y)=\sup \left\{u(x): u \in \operatorname{CVX}(\Omega), u \leq 0, M u \geq \delta_{y}\right\} .
$$

We may therefore treat $g_{\Omega}$ as a real counterpart of the pluricomplex Green function defined by Klimek [10]. Demailly [5] proved that the Green function can be regarded as a fundamental solution for the complex Monge-Ampère operator $M_{c}$ in the class of hyperconvex domains in $C^{n}$ and it is continuous there. In section 1 we show this is also the case with $g_{\Omega}$ (Theorem 1.5). We use a method going back to Walsh [14]. The next problem is the symmetry. As follows from a deep results of Lempert [12] the pluricomplex Green function is symmetric in convex domains in $\mathrm{C}^{n}$ (see also [8]). On the other hand it need not be symmetric in strictly pseudoconvex domains as shown in [1]. Somewhat to our surprise it turned out that $g_{\Omega}$ is never symmetric except the one dimensional case (Theorem 3.1). Finally, in Theorem 4.1 we give a formula for the fundamental solutions in product domains. The corresponding results in the complex case can be found in [8], [9] and [6].

In fact, similarly as in the complex case, we could use $(0.3)$ as a definition of $g_{\Omega}$ and it would make sense also for nonconvex $\Omega$. The following example shows that in such a case it is not true in general that $g_{\Omega}$ is a restriction of $g_{\widehat{\Omega}}$, where $\widehat{\Omega}$ is the convex hull of $\Omega$ : let $\Omega$ be equal to the unit disc $B$ in $\mathrm{R}^{2}$ minus the triangle with vertices at, say, $(1 / 3,1 / 3),(1 / 3,-1 / 3)$ and $(0,1 / 2)$. Then $\widehat{\Omega}=B$ and one can prove that on the triangle with vertices at $(1 / 3,1 / 3),(1 / 3,-1 / 3)$ and $(0,0) g_{\Omega}(\cdot, 0)$ is affine whereas $g_{B}(\cdot, 0)$ is not. A corresponding example in the complex case can be found in [8], Exercise 5.8.

A few of the results presented below are not new but we include brief proofs of them in order to be complete. This applies to Proposition 1.1 (its proof is essentially included in the proof of Lemma 3.5 in [13]), Proposition 3.2 (see for example [7], pp. 23-24) and Theorem 3.4 which can be essentially found in [4].

The paper was written during the first author's stay at the Mid Sweden University in Sundsvall. He is grateful for the hospitality he received there.

\section{Continuity.}

Throughout this section we assume that $\Omega$ is a bounded, convex domain in $\mathrm{R}^{n}$ and write $d_{\Omega}(x):=\operatorname{dist}(x, \partial \Omega)$. 
Proposition 1.1. For $y \in \Omega$ we have

$$
c_{n} d_{\Omega}(y) \leq\left|h_{\Omega}(y)\right| \leq d_{n}(\operatorname{diam} \Omega)^{1-1 / n} d_{\Omega}(y)^{1 / n},
$$

where $c_{n}$ and $d_{n}$ are positive constants depending only on $n$.

Proof. Let $A$ denote the gradient image of the function $u:=\left|h_{\Omega}(y)\right|^{-1} g_{\Omega}(\cdot, y)$ and set $r:=\operatorname{dist}(y, \partial \Omega), R:=\operatorname{diam} \Omega$. In fact, the graph of $u$ is a cone with basis $\bar{\Omega} \times\{0\}$ with vertex at $(y,-1)$. One can show that $A$ is a convex set containing at least one vector of length $1 / r$ (take a supporting hyperplane to the epigraph of $u$ along the line segment joining $y$ with the closest point from $\partial \Omega$ ). Moreover

$$
\bar{B}(0,1 / R) \subset A \subset \bar{B}(0,1 / r) .
$$

Therefore

$$
\frac{d_{n}^{\prime}}{R^{n-1} r} \leq \lambda(A) \leq \frac{c_{n}^{\prime}}{r^{n}}
$$

where $c_{n}^{\prime}$ and $d_{n}^{\prime}$ are positive constants depending only on $n$. Now the proposition follows since $\lambda(A)=\left|h_{\Omega}(y)\right|^{-n}$.

Proposition 1.1 implies in particular that $\lim _{y \rightarrow \partial \Omega} h_{\Omega}(y)=0$. Hence it makes sense to set $g_{\Omega}(x, y):=0$ if $y \in \partial \Omega$

Proposition 1.2. For $x, y \in \Omega$ we have

$$
\left|h_{\Omega}(y)\right|\left(1-\frac{|x-y|}{d_{\Omega}(y)}\right) \leq\left|g_{\Omega}(x, y)\right| \leq \min \left\{\left|h_{\Omega}(x)\right|,\left|h_{\Omega}(y)\right|\right\} .
$$

Proof. For every $x, y \in \Omega$ one can find $P \in \partial \Omega$ such that $x$ belongs to the line segment joining $y$ and $P$. Then

$$
g_{\Omega}(x, y)=h_{\Omega}(y) \frac{|x-P|}{|y-P|}=h_{\Omega}(y)\left(1-\frac{|x-y|}{|y-P|}\right) .
$$

This proves the first inequality. It remains to show that $\left|g_{\Omega}(x, y)\right| \leq\left|h_{\Omega}(x)\right|$. To prove it assume that $g_{\Omega}(x, y) \leq A h_{\Omega}(x)$ for some $x, y \in \Omega, A>1$ and set $u:=g_{\Omega}(\cdot, y), v:=A g_{\Omega}(\cdot, x)$. Since $u=v$ on $\partial \Omega, u$ is convex, $v$ is affine on line segments joining $x$ with the boundary and $u(x) \leq v(x)$, it follows that $u \leq v$ in $\Omega$. It is easy to see that the gradient image of $u$ contains the gradient image of $v$ (see [13], Proposition 2.7) and therefore

$$
1=\int_{\Omega} M g_{\Omega}(\cdot, y) \geq \int_{\Omega} M\left(A g_{\Omega}(\cdot, x)\right)=A^{n}
$$

which is a contradiction. 
Corollary 1.3. For $y \in \bar{\Omega}, z \in \partial \Omega$ and $0 \leq t \leq 1$ we have

$$
h_{\Omega}(t y+(1-t) z) \leq t h_{\Omega}(y) .
$$

Proof. Proposition 1.2 gives

$$
h_{\Omega}(t y+(1-t) z) \leq g_{\Omega}(t y+(1-t) z, y)=t h_{\Omega}(y) .
$$

THEOREM 1.4. $h_{\Omega}$ is locally Hölder continuous in $\Omega$ with exponent $1 / n$.

Proof. Fix $w \in \Omega$ and set $r:=d_{\Omega}(w)$. Take $y, z \in B(w, \varepsilon)$, where $0<\varepsilon<r$ will be determined later. Proposition 1.1 gives

$$
\left|h_{\Omega}(x)\right| \leq C d_{\Omega}(x)^{1 / n}, \quad x \in \bar{\Omega},
$$

where $C:=d_{n}(\operatorname{diam} \Omega)^{1-1 / n}$. For $x \in \bar{\Omega}$ define

$$
u(x):= \begin{cases}\max \left\{a g_{\Omega}(x+y-z, y)-C|y-z|^{1 / n}, g_{\Omega}(x, y)\right\} & \text { if } x+y-z \in \bar{\Omega}, \\ g_{\Omega}(x, y) & \text { if } x+y-z \notin \bar{\Omega},\end{cases}
$$

where $a>0$ will be determined later. If $x+y-z \in \partial \Omega$ then $d_{\Omega}(x) \leq|y-z|$ and from Proposition 1.2 and (1.2) it follows that

$$
g_{\Omega}(x, y) \geq h_{\Omega}(x) \geq-C|y-z|^{1 / n}=a g_{\Omega}(x+y-z, y)-C|y-z|^{1 / n} .
$$

This implies that $u$ is convex in $\Omega$.

Now we want to find $a>0$ so that

$$
u(x)=a g_{\Omega}(x+y-z, y)-C|y-z|^{1 / n} \text { for } x \text { near } z .
$$

(1.3) will be satisfied exactly if $a h_{\Omega}(y)-C|y-z|^{1 / n}>g_{\Omega}(y, z)$. From Propositions 1.1 and 1.2 we infer

$$
\begin{aligned}
\frac{\left|g_{\Omega}(y, z)\right|-C|y-z|^{1 / n}}{\left|h_{\Omega}(y)\right|} & \geq \frac{c_{n}\left(d_{\Omega}(y)-|y-z|\right)-C|y-z|^{1 / n}}{C d_{\Omega}(y)^{\frac{1}{n}}} \\
& \geq \frac{c_{n}(r-3 \varepsilon)-C(2 \varepsilon)^{1 / n}}{C(r+\varepsilon)^{1 / n}}=: Q .
\end{aligned}
$$

If we choose $0<\varepsilon<r$ so that $Q>0$ and $a>0$ so that $a<Q$ then we get (1.3). This implies that $M(u / a) \geq \delta_{z}$ and by the comparison principle $u / a \leq g_{\Omega}(\cdot, z)$. Since $u(z) \geq a h_{\Omega}(y)-C|y-z|^{1 / n}$ we arrive at the estimate

$$
h_{\Omega}(y)-h_{\Omega}(z) \leq \frac{C}{a}|y-z|^{1 / n}
$$

which concludes the proof of Theorem 1.4. 
We conjecture that $h_{\Omega}$ is convex and smooth in $\Omega$.

THEOREM 1.5. $g_{\Omega}$ is continuous on $\bar{\Omega} \times \bar{\Omega}$

Proof. By Propositions 1.1 and 1.2 it is enough to prove the continuity on $\Omega \times \Omega$. For $x, y \in \Omega, x \neq y$, let $P=P(x, y)$ be as in the proof of Proposition 1.2. One can show that $P$ is continuous on $\Omega \times \Omega \backslash \Delta$, where $\Delta$ is the diagonal of $\Omega \times \Omega$. Since $h_{\Omega}$ is continuous, (1.1) shows immidiately that $g_{\Omega}$ is continuous on $\Omega \times \Omega \backslash \Delta$. To show that it is also continuous at some $\left(y_{0}, y_{0}\right) \in \Delta$ write

$$
\begin{aligned}
\left|g_{\Omega}(x, y)-g_{\Omega}\left(y_{0}, y_{0}\right)\right| & =\left|h_{\Omega}(y)\left(1-\frac{|x-y|}{|y-P|}\right)-h_{\Omega}\left(y_{0}\right)\right| \\
& \leq\left|h_{\Omega}(y)-h_{\Omega}\left(y_{0}\right)\right|+\left|h_{\Omega}(y)\right| \frac{|x-y|}{\operatorname{dist}(y, \partial \Omega)}
\end{aligned}
$$

and the right hand-side obviously converges to zero as $x$ and $y$ tend to $y_{0}$.

\section{The fundamental solution in the unit ball.}

In this section we want to compute $h_{B}$, where $B$ is the unit ball in $\mathrm{R}^{n}$.

Lemma 2.1. Let $y \in B$ and set $u:=\left|h_{B}(y)\right|^{-1} g_{B}(\cdot, y)$. Then for $x \in \partial B$ we have

$$
\nabla u(x)=\frac{x}{1-\langle x, y\rangle} .
$$

(u extends naturally to a convex function in $\mathrm{R}^{n}$, smooth away from the origin.)

Proof. By $T_{x}$ denote the tangent hyperplane to $\partial B$ at $x$. Then the supporting function of $u$ at $x$ (that is an affine function whose graph is a supporting hyperplane for the epigraph of $u$ at $x$ ) vanishes on $T_{x}$ and the length of its gradient is equal to $1 / \operatorname{dist}\left(y, T_{x}\right)$. Therefore

$$
\nabla u(x)=\frac{x}{\operatorname{dist}\left(y, T_{x}\right)}=\frac{x}{\left|x^{*}-y\right|}
$$

for some $x^{*} \in T_{x}$. The vector $x$ is perpendicular to $T_{x}$, thus $x^{*}=y+\alpha x$ for some $\alpha>0$. Since $\left\langle x^{*}-x, x\right\rangle=0$ it follows that $\left|x^{*}-y\right|=\alpha=1-\langle x, y\rangle$.

Lemma 2.2. Take $0 \leq a<1, y:=(0, \ldots, 0, a)$ and let $u$ be as in Lemma 2.1. Then the gradient image of $u$ is the set of all $w=\left(w^{\prime}, w_{n}\right) \in \mathbf{R}^{n}$ with

$$
\frac{\left|w^{\prime}\right|^{2}}{1-a^{2}}+\left(w_{n}-\frac{a}{1-a^{2}}\right)^{2} \leq \frac{1}{\left(1-a^{2}\right)^{2}} \text {. }
$$


PRoof. The boundary of the gradient image of $u$ is equal to the image of $\partial B$ by $\nabla u$. For $x=\left(x^{\prime}, x_{n}\right) \in \partial B$ let $w:=\nabla u(x)=x /(1-\langle x, y\rangle)=$ $x /\left(1-a x_{n}\right)$ (by Lemma 2.1). Then

$$
\begin{aligned}
\frac{\left|w^{\prime}\right|^{2}}{1-a^{2}}+\left(w_{n}-\frac{a}{1-a^{2}}\right)^{2} & =\frac{\left|x^{\prime}\right|^{2}}{\left(1-a^{2}\right)\left(1-a x_{n}\right)^{2}}+\left(\frac{x_{n}}{1-a x_{n}}-\frac{a}{1-a^{2}}\right)^{2} \\
& =\frac{\left(1-a^{2}\right)\left(1-x_{n}^{2}\right)+\left(x_{n}\left(1-a^{2}\right)-a\left(1-a x_{n}\right)\right)^{2}}{\left(1-a^{2}\right)^{2}\left(1-a x_{n}\right)^{2}} \\
& =\frac{1}{\left(1-a^{2}\right)^{2}}
\end{aligned}
$$

which completes the proof.

Theorem 2.3. For $y \in B$ we have

$$
h_{B}(y)=-b_{n}^{-1 / n}\left(1-|y|^{2}\right)^{(n+1) / 2 n},
$$

where $b_{n}$ is the volume of $B$.

Proof. Let $u$ be as in Lemma 2.1. From Lemma 2.2 it follows that the volume of the gradient image of $u$ is equal to $b_{n}\left(1-|y|^{2}\right)^{-(n+1) / 2}$. Therefore

$$
1=\int_{B} M g_{B}(\cdot, y)=\left|h_{B}(y)\right|^{n} \int_{B} M u=b_{n}\left(1-|y|^{2}\right)^{-(n+1) / 2}\left|h_{B}(y)\right|^{n}
$$

and the theorem follows.

\section{Symmetry.}

The goal of this section is to prove the following

TheOREM 3.1. Let $\Omega$ be a nonempty, bounded, convex domain in $\mathrm{R}^{n}$. Then $g_{\Omega}$ is symmetric if and only if $n=1$.

Before proving Theorem 3.1 we need some auxiliary results. First consider the one dimensional case. Let $I=(a, b)$ be an interval in $\mathrm{R}$. We will say that a function $g: I \times I \longrightarrow \mathrm{R}$ is an $x$-cone if for every $y \in I$ the function $g(\cdot, y)$ is continuous on $\bar{I}$, affine on the intervals $[a, y],[y, b]$ and $g(a, y)=g(b, y)=0$. It is obvious that if two $x$-cones are such that for every $y \in I$ there exists $x \in I$ such that they are equal at $(x, y)$ then they are equal everywhere. 
Proposition 3.2. We have

$$
g_{I}(x, y)= \begin{cases}\frac{(x-a)(y-b)}{b-a} & \text { if } a \leq x \leq y \leq b, \\ \frac{(y-a)(x-b)}{b-a} & \text { if } a \leq y \leq x \leq b .\end{cases}
$$

In particular $g_{I}$ is symmetric.

Proof. Fix $y \in I$. It is easy to see that the gradient image of the function $\left|h_{I}(y)\right|^{-1} g_{I}(\cdot, y)$ is equal to $\left[(a-y)^{-1},(b-y)^{-1}\right]$. Therefore

$$
1=\int_{I} \operatorname{Mg}_{I}(\cdot, y)=\left|h_{I}(y)\right| \frac{a-b}{(y-a)(y-b)}
$$

and

$$
h_{I}(y)=\frac{(y-a)(y-b)}{b-a} .
$$

Now the proposition follows since both sides of (3.1) are $x$-cones equal on the diagonal of $I \times I$.

Proposition 3.3. For an $x$-cone $g$ the following are equivalent

i) $g$ is symmetric;

ii) $g(y, y)$ is a quadratic polynomial vanishing at a and $b$;

iii) $g=c g_{I}$ for some constant $c$.

Proof. The implications iii) $\Rightarrow$ i) and iii) $\Rightarrow$ ii) follow from Proposition 3.2. To show the converse ones it is enough to observe that in cases i) and ii) all values of $g$ are determined by the value of $g$ at one point $\left(y_{0}, y_{0}\right) \in I \times I$ from the diagonal.

We will also need the following result which says that separate polynomials are polynomials (see [4]).

Theorem 3.4. Let $h$ be a function defined on a domain $D$ in $\mathrm{R}^{n}$ such that for every $x=\left(x_{1}, \ldots, x_{n}\right) \in D$ and for every $i=1, \ldots, n$ the function $h\left(x_{1}, \ldots, x_{i-1}, \cdot, x_{i+1}, \ldots, x_{n}\right)$ is a restriction of a polynomial of degree $\leq d$. Then $h$ is a restriction of a polynomial in $\mathrm{R}^{n}$ of degree $\leq d$.

Proof. The theorem will easily follow from the following fact which can be proved by means of the Lagrange interpolation: if each of the sets $A_{i} \subset \mathrm{R}$, $i=1, \ldots, n$, consists of exactly $d+1$ elements and $A=A_{1} \times \ldots \times A_{n}$ then the mapping 


$$
\begin{aligned}
& \left\{\text { polynomials in } \mathrm{R}^{n} \text { of degree } \leq d\right\} \ni \\
& \left.p \longmapsto p\right|_{A} \in\{\text { functions from } A \text { to } \mathrm{R}\}
\end{aligned}
$$

is bijective. For we may assume $D$ is a cube $\left(a_{1}, b_{1}\right) \times \ldots \times\left(a_{n}, b_{n}\right)$ and take $A_{i} \subset\left(a_{i}, b_{i}\right), i=1, \ldots, n$ as above. By the inductive arguments we may assume that $h$ is a polynomial in first $n-1$ variables. By the surjectivity of the mapping (3.2) there is a polynomial $p$ such that $p=h$ on $A$. From the injectivity of the mapping in $\mathrm{R}$ it follows that $p=h$ on $A_{1} \times \ldots \times A_{n-1} \times\left(a_{n}, b_{n}\right)$ and from the injectivity in $\mathrm{R}^{n-1}$ we get $p=h$ on $D$.

Proof of Theorem 3.1. Assume that $g_{\Omega}$ is symmetric. From Proposition 3.3 it follows that $h_{\Omega}$ is a quadratic polynomial on every line segment contained in $\Omega$. By Theorem $3.4 h_{\Omega}$ is a restriction of a quadratic polynomial $p$ in $\mathbf{R}^{n}$ and thus $\Omega=\{p<0\}$. Since $\Omega$ is bounded it follows that there is an affine isomorphism $L$ of $\mathrm{R}^{n}$ mapping $\Omega$ onto the unit ball $B$. It is well-known and easy to check that for every convex function $u$ in $B$ one has

$$
M(u \circ L)=|\operatorname{det} L|^{2} L^{*} M u,
$$

where $L^{*} M u$ is the pullback of the measure $M u$ by $L$. Since $L^{*} \delta_{L(y)}=|\operatorname{det} L|^{-1} \delta_{y}$ it follows that $g_{\Omega}=|\operatorname{det} L|^{-1 / n} g_{B} \circ(L, L)$. Therefore $h_{B}$ is a quadratic polynomial and from Theorem 2.3 it follows that $n=1$.

\section{The product property.}

In this section we want to prove the following

THeORem 4.1. If $\Omega_{i}$ is a bounded convex domain in $\mathrm{R}^{n_{i}}, i=1,2$, then for $x_{i}, y_{i} \in \Omega_{i}$ we have

$$
h_{\Omega_{1} \times \Omega_{2}}\left(y_{1}, y_{2}\right)=-\left(\begin{array}{c}
n_{1}+n_{2} \\
n_{1}
\end{array}\right)^{1 /\left(n_{1}+n_{2}\right)}\left|h_{\Omega_{1}}\left(y_{1}\right)\right|^{n_{1} /\left(n_{1}+n_{2}\right)}\left|h_{\Omega_{2}}\left(y_{2}\right)\right|^{n_{2} /\left(n_{1}+n_{2}\right)}
$$

and

$$
\begin{aligned}
& g_{\Omega_{1} \times \Omega_{2}}\left(\left(x_{1}, x_{2}\right),\left(y_{1}, y_{2}\right)\right) \\
& =\left|h_{\Omega_{1} \times \Omega_{2}}\left(y_{1}, y_{2}\right)\right| \max \left\{\left|h_{\Omega_{1}}\left(y_{1}\right)\right|^{-1} g_{\Omega_{1}}\left(x_{1}, y_{1}\right),\left|h_{\Omega_{2}}\left(y_{2}\right)\right|^{-1} g_{\Omega_{2}}\left(x_{2}, y_{2}\right)\right\} .
\end{aligned}
$$

Proof. The second equation follows from the fact that both functions are affine along line segments joining $\left(y_{1}, y_{2}\right)$ with $\partial\left(\Omega_{1} \times \Omega_{2}\right)$ and equal at the endpoints. It remains to prove the first one. By $E_{i}, i=1,2$, denote the gradient image of $\left|h_{\Omega_{i}}\left(y_{i}\right)\right|^{-1} g_{\Omega_{i}}\left(\cdot, y_{i}\right)$ and by $E$ the gradient image of $\left|h_{\Omega_{1} \times \Omega_{2}}\left(y_{1}, y_{2}\right)\right|^{-1} g_{\Omega_{1} \times \Omega_{2}}\left(\cdot,\left(y_{1}, y_{2}\right)\right)$. From the definition of a gradient image it follows that 


$$
E_{i}=\left\{p_{i} \in \mathrm{R}^{n_{i}}: s_{i}\left(p_{i}\right):=\max _{x_{i} \in \bar{\Omega}_{i}}\left\langle x_{i}-y_{i}, p_{i}\right\rangle \leq 1\right\}
$$

and

$$
E=\left\{\left(p_{1}, p_{2}\right) \in \mathrm{R}^{n_{1}+n_{2}}: s_{1}\left(p_{1}\right)+s_{2}\left(p_{2}\right) \leq 1\right\} .
$$

(In fact, one can show that $E$ is exactly the convex hull of the set $E_{1} \times\{0\} \cup\{0\} \times E_{2}$, but we will not need this observation later.) By the Fubini theorem and since $s_{1}$ and $s_{2}$ are homogeneous functions, we have

$$
\begin{aligned}
\lambda_{n_{1}+n_{2}}(E) & =\int_{E_{1}} \lambda_{n_{2}}\left(\left\{p_{2} \in \mathrm{R}^{n_{2}}: s_{1}\left(p_{1}\right)+s_{2}\left(p_{2}\right) \leq 1\right\}\right) d \lambda_{n_{1}}\left(p_{1}\right) \\
& =\int_{E_{1}} \lambda_{n_{2}}\left(\left(1-s_{1}\left(p_{1}\right)\right) E_{2}\right) d \lambda_{n_{1}}\left(p_{1}\right) \\
& =\lambda_{n_{2}}\left(E_{2}\right) \int_{E_{1}}\left(1-s_{1}\left(p_{1}\right)\right)^{n_{2}} d \lambda_{n_{1}}\left(p_{1}\right) \\
& =\lambda_{n_{2}}\left(E_{2}\right) \lambda_{n_{1}+1}\left(\left\{\left(p_{1}, t\right) \in E_{1} \times[0,1]: t \leq\left(1-s_{1}\left(p_{1}\right)\right)^{n_{2}}\right\}\right) \\
& =\lambda_{n_{2}}\left(E_{2}\right) \int_{0}^{1} \lambda_{n_{1}}\left(\left(1-t^{1 / n_{2}}\right) E_{1}\right) d t \\
& =\lambda_{n_{1}}\left(E_{1}\right) \lambda_{n_{2}}\left(E_{2}\right) \int_{0}^{1}\left(1-t^{1 / n_{2}}\right)^{n_{1}} d t .
\end{aligned}
$$

One can show that

$$
\int_{0}^{1}\left(1-t^{1 / n_{2}}\right)^{n_{1}} d t=\int_{0}^{1} n_{2} t^{n_{2}-1}(1-t)^{n_{1}} d t=\frac{n_{1} ! n_{2} !}{\left(n_{1}+n_{2}\right) !} .
$$

We have $\left|h_{\Omega_{i}}\left(y_{i}\right)\right|=\lambda_{n_{i}}\left(E_{i}\right)^{-1 / n_{i}}$ and $\left|h_{\Omega_{1} \times \Omega_{2}}\left(y_{1}, y_{2}\right)\right|=\lambda_{n_{1}+n_{2}}(E)^{-1 /\left(n_{1}+n_{2}\right)}$ and this completes the proof.

Added In Proof. In the article Z. Blocki, Regularity of the fundamental solution for the Monge-Ampère operator, Pitman Res. Notes Math. Ser. 383, Progress in partial differential equations, Pont-à-Mousson 1997, 1 (1998), $40-45$, it was proved in particular that $h_{\Omega}$ is always smooth and convex. 


\section{REFERENCES}

1. E. Bedford, J.-P. Demailly, Two counter examples concerning the pluri-complex Green function in $\mathrm{C}^{n}$, Indiana Univ. Math. J.37 (1988), 865-867.

2. E. Bedford, B. A. Taylor, The Dirichlet problem for a complex Monge-Ampère equation. Invent. Math. 37 (1976), 1-44.

3. Z. Błocki, Smooth exhaustion functions in convex domains, Proc. Amer. Math. Soc. 125 (1997), 477-484.

4. J. Bochnak, J. Siciak, Polynomials and multilinear mappings in topological vector spaces, Studia Math. 39 (1971), 59-76

5. J.-P. Demailly, Mesures de Monge-Ampère et mesures plurisousharmoniques, Math. Z. 194 (1987), 519-564.

6. A. Edigarian, On the product property of the pluricomplexGreen function, Proc. Amer. Math. Soc. 125 (1997), 2855-2858.

7. L. Hörmander, Notions of Convexity, Birkhäuser, 1994.

8. M. Jarnicki, P. Pflug, Invariant Distances and Metrics in Complex Analysis, Walter de Gruyter, 1993.

9. M. Jarnicki, P. Pflug, Remarks on the pluricomplex Green function Indiana Univ. Math. J. 44 (1995), 535-543.

10. M. Klimek, Extremal plurisubharmonic functions and invariant pseudodistances, Bull. Soc. Math. Fr. 113 (1985), 231-240.

11. M. Klimek, Pluripotential Theory, Clarendon Press,1991.

12. L. Lempert, La métrique de Kobayashi et la représentation des domaines sur la boulle, Bull. Soc. Math. Fr.109 (1981), 427-474.

13. J. Rauch, B. A. Taylor, The Dirichlet problem for the multidimensional Monge-Ampère equation, Rocky Mountain Math. J. 7 (1977), 345-364.

14. J.B. Walsh, Continuity of envelopes of plurisubharmonic functions, J. Math. Mech. 18 (1968), 143-148.

JAGIELLONIAN UNIVERSITY INSTITUTE OF MATHEMATICS

REYMONTA 4

30-059 KRAKÓW

POLAND

e-mail: blocki@im.uj.edu.pl
MID SWEDEN UNIVERSITY

FMI

85170 SUNDSVALL

SWEDEN

e-mail: johant@fmi.mh.se 